\title{
Estresse ocupacional e cultura de segurança: tendências para contribuição e construção do conhecimento em enfermagem
}

\author{
Occupational stress and safety culture: trends for \\ contribution and construction of knowledge in nursing \\ Oclaris Lopes Munhoz¹, Rafaela Andolhe², Maria Denise Schimith², Gabriela Oliveira'1, Polyana de Lima Ribeiro' \\ Thayná Champe da Silva ${ }^{1}$, Luiza Cremonese \\ ${ }^{1}$ Programa de Pós-Graduação em Enfermagem, Universidade Federal de Santa Maria - (UFSM) - Santa Maria (RS), Brasil. \\ ${ }^{2}$ Departamento de Enfermagem, (UFSM) - Santa Maria (RS), Brasil.
}

DOI: http://dx.doi.org/10.7322/abcshs.v43i2.991

\begin{abstract}
RESUMO
O estresse é considerado um dos fatores que mais atinge os profissionais de enfermagem, podendo repercutir na cultura de segurança e desfavorecer a assistência prestada. O objetivo do presente estudo foi identificar e caracterizar as tendências das teses e dissertações em enfermagem produzidas no Brasil, acerca da temática do estresse ocupacional da equipe de enfermagem associado à cultura de segurança. Trata-se de um estudo de revisão narrativa. A busca foi realizada no portal de teses e dissertações da CAPES, no mês de maio de 2017, sem recorte temporal. Houve predominância de dissertações, com abordagem quantitativa, no ano de 2015, na região sul. A fragilidade da cultura de segurança foi identificada nos estudos voltados à segurança do paciente. Os resultados apontaram, ainda, fragilidades nas ações desenvolvidas e a necessidade de implantar estratégias que colaborassem para uma melhor assistência em saúde. Ainda, o estresse ocupacional foi reconhecido, por parte dos profissionais como influência negativa no trabalho. Desvendou-se, também, como uma tendência das produções, a predominância de estudos de cultura de segurança em unidades hospitalares fechadas.
\end{abstract}

Palavras-chave: estresse ocupacional; cultura organizacional; segurança do paciente.

\begin{abstract}
Stress is considered to be one of the factors that affect nursing professionals the most and may disturb the safety culture and disfavor the assistance provided. The objective of the present study was to identify and characterize the tendencies of the theses and dissertations in nursing produced in Brazil about the subject of occupational stress of the nursing team associated to the safety culture. It is a narrative review study. The search was carried out in the portal of theses and dissertations of CAPES, in May 2017, without temporal cut. There was a predominance of dissertations, with a quantitative approach, in the year 2015, in the southern region. The fragility of the safety culture has been identified in studies regarding patient safety. The results also pointed to weaknesses in the actions developed and the need to implement strategies that would contribute to better health care. Still, occupational stress was recognized by professionals as a negative influence on work. Therefore, a predominance of safety culture studies in closed hospital units was revealed as a trend of production.
\end{abstract}

Keywords: occupational stress; organizational culture; patient safety. 


\section{INTRODUÇÃO}

Aliada ao avanço na assistência em saúde, a segurança do paciente passou a ser uma das maiores preocupações dos órgãos e instituições de saúde em nível mundial. Essa preocupação iniciou ainda no século XX, com a publicação do relatório do Institute of Medicine dos Estados Unidos da América, que divulgou dados alarmantes referentes a erros na assistência em saúde ${ }^{1}$.

No que diz respeito ao ambiente de trabalho e à cultura de segurança, deve-se levar em consideração as ações dos profissionais de saúde frente às suas práticas. Assim, a cultura de segurança provém da cultura organizacional, entendida como o conjunto de valores, normas, crenças e expectativas presentes entre os participantes de uma determinada organização. Neste contexto, o clima organizacional, uma vez mensurado, pode ser considerado como indicador de comportamento, produtividade, motivação e satisfação dos profissionais ${ }^{2}$.

Segundo Karasek ${ }^{3}$, o estresse ocupacional é definido como a relação entre altas demandas psicológicas, baixo controle sobre o trabalho e baixo apoio social recebido no ambiente laboral. Com repercussão na segurança do paciente, o estresse ocupacional é considerado um dos fatores que mais atinge os profissionais de enfermagem e, consequentemente, influencia na atuação da equipe de enfermagem na prestação dos serviços de saúde, gerando insegurança na prestação da assistência ${ }^{4}$.

A equipe de enfermagem, principalmente hospitalar, acompanha permanentemente os pacientes durante as 24 horas e está exposta a diversos estressores. Executa diversas atividades, tais como o atendimento individual e coletivo, o relacionamento interpessoal e multiprofissional; atua em situações de urgência e emergência; lida com a falta de recursos humanos e materiais, o que resulta em carga de trabalho excessiva, sendo que estes fatores podem contribuir para o desenvolvimento do estresse ocupacional, o que pode pôr em risco a segurança dos pacientes que dependem destes profissionais ${ }^{5}$.

Um estudo realizado em uma instituição hospitalar identificou que os enfermeiros reconheceram que os estressores do ambiente de trabalho podiam influenciar no desempenho da assistência prestada. Dentre os estressores identificados, estavam a carga de trabalho excessiva e a insatisfação profissional. Com isso, os profissionais trabalhavam com descuidos, ocasionando erros. Nesta perspectiva, percebe-se que o estresse ocupacional tem influência direta na cultura de segurança ${ }^{6}$.

Diante do exposto, o estudo justifica-se na medida em que a enfermagem também é responsável pela cultura de segurança, bem como é atingida pelos estressores do seu ambiente de trabalho ${ }^{3-6}$. Frente às essas afirmações e devido ao aumento das pesquisas a respeito do estresse e da cultura de segurança e a necessidade de conhecimento e aprimoramento dos profissionais da saúde, acerca da temática, o presente estudo teve como questão-guia: qual a tendência das teses e dissertações defendidas pelos Programas de Pós-Graduação em Enfermagem do Brasil sobre o estresse ocupacional da equipe de enfermagem associado à cultura de segurança?
Entendeu-se que analisar o estresse ocupacional da equipe de enfermagem associado à cultura de segurança, possibilitou o planejamento de estratégias favoráveis à saúde do trabalhador e à segurança do paciente. Buscando responder à questão de pesquisa, elencou-se como objetivo geral, identificar e caracterizar as tendências das teses e dissertações em enfermagem produzidas no Brasil, acerca da temática do estresse ocupacional da equipe de enfermagem associado à cultura de segurança.

\section{MÉTODOS}

Tratou-se de um estudo de revisão bibliográfica, do tipo narrativo, baseado nas tendências nacionais de teses e dissertações acerca da temática descrita. Para a obtenção das publicações foi realizada uma busca no Banco de Teses e Dissertações da Coordenação de Aperfeiçoamento de Pessoal de Nível Superior (CAPES), no mês de maio de 2017. As revisões narrativas são consideradas estudos qualitativos e, que possuem como foco, a descrição e a discussão, de forma ampla, de um determinado assunto, ainda, podendo apresentar uma análise crítica e pessoal do pesquisador?

Como estratégia de busca avançada no Banco de Dissertações e Teses da CAPES, utilizou-se a seguinte palavra-chave: "cultura de segurança”, sem restrição de área do conhecimento. Dessa forma, foram encontradas 197 produções, sendo que, após, foi realizada a leitura de título, buscando-se também, a palavra estresse. Cabe destacar que a busca pela palavra estresse aconteceu como uma estratégia para captar maior número de produções, pois alguns estudos abordavam o estresse ocupacional sem nomeá-lo como tal.

Como critérios de inclusão definiu-se que seriam selecionados estudos acadêmicos nacionais, oriundos de Programas de Pós-Graduação em Enfermagem, que abordassem a temática do estresse ocupacional associada à cultura de segurança, como resultados de teses e dissertações, e sem recorte temporal, o que permitiu identificar a tendência das produções na referida temática. Os critérios de exclusão compreenderam estudos que possuíam resumos incompletos ou indisponíveis e estudos metodológicos que envolvessem construção, validação e/ou adaptação de instrumentos. Cabe mencionar que não foram encontrados estudos duplicados. Após a aplicação dos critérios supracitados e leitura das produções, constituiu-se o corpus de 27 estudos científicos para a análise, conforme a Figura 1.

Com o objetivo de organizar e compreender a análise dos dados foi elaborado um quadro sinóptico, composto pelas seguintes informações: título, autor (a), ano, nível do estudo (tese ou dissertação) e instituição. Todos os estudos selecionados foram expostos no Quadro 1.

Os dados foram interpretados por uma abordagem qualitativa e com análise crítica pessoal, apresentados por meio de duas categorias, visto que se tratou de uma revisão narrativa.

No que se referiu aos aspectos éticos em pesquisa, as definições e resultados usados pelos autores dos estudos selecionados para a análise foram reproduzidos fidedignamente e sem sofrer alterações. 


\section{RESULTADOS E DISCUSSÃO}

A partir da leitura, análise e caracterização dos estudos selecionados e da extração dos dados disponibilizados nos respectivos estudos $^{8-34}$, foi possível evidenciar as tendências da produção na enfermagem brasileira, a respeito da temática do estresse ocupacional da equipe de enfermagem associado à cultura de segurança, bem como apresentar categorias para a análise.

\section{Caracterização das produções relacionadas ao estresse e à cultura de segurança}

Conforme o Quadro 1, das vinte e sete produções selecionadas, 9 (33,3\%) foram publicadas em 2015; 7 (25,9\%), em 2013; $6(22,2 \%)$, em 2014 e $5(18,6 \%)$ no ano de 2016 , com uma média de 6,75 pesquisas por ano. Destas, vinte e três $(85,2 \%)$ eram dissertações (mestrado acadêmico) e $4(14,8 \%)$ teses. Quanto à área do conhecimento, 21 (77,8\%) eram da enfermagem ${ }^{8-19,21-28,34}$; $2(7,4 \%)$ correspondiam à enfermagem fundamental ${ }^{32,33} ; 1(3,7 \%)$, à atenção à saúde ${ }^{20} ; 1(3,7 \%)$, à enfermagem na saúde do adulto ${ }^{29}$; $1(3.7 \%)$, às ciências para a saúde ${ }^{30}$ e $1(3.7 \%)$ referente à área de saúde, sociedade e ambiente ${ }^{31}$.

Em relação à região geográfica das pesquisas selecionadas, a predominância foi na região sul, com doze (44,5\%) estudos. Dentre as universidades que faziam parte da referida região, 8 estudos foram desenvolvidos pelos Programas de Pós-Graduação em Enfermagem da Universidade Federal de Santa Catariana (UFSC); 1 na Universidade Federal de Rio Grande (FURG); 1 na Universidade do Vale do Rio dos Sinos (UNISINOS); 1 na Universidade Federal de Santa Maria (UFSM) e 1 na Universidade

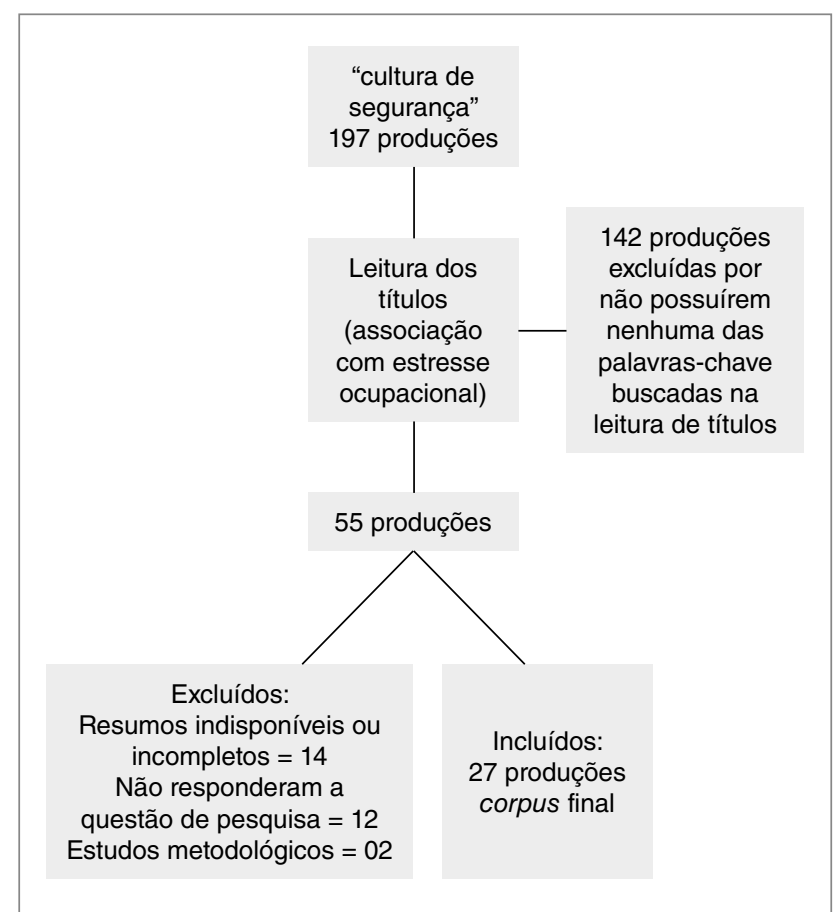

Figura 1: Fluxograma do corpus das produções selecionadas. Santa Maria (RS), 2017.
Federal do Paraná (UFPR). Na sequência, apareceu a região sudeste, com 10 (37\%) estudos, destes, 4 foram desenvolvidos na Universidade de São Paulo (USP); 1 na Universidade Estadual de Campinas (UNICAMP); 1 na Universidade Estadual do Rio de Janeiro (UERJ); 1 na Universidade Federal do Estado do Rio de Janeiro (UNIRIO); 1 na Universidade Federal dos Vales do Jequitinhonha e do Mucuri (UFVJM); 1 na Universidade Federal do Espírito Santo (UFES) e 1 na Universidade Federal do Triângulo Mineiro (UFTM). Após, apareceu a região centro-oeste, com 3 (11,1 \%) produções; 2 na Universidade Federal de Goiás (UFG) e 1 na Escola Superior de Ciências da Saúde (FEPECS). Por fim, apareceu a região nordeste, com $2(7,4 \%)$ tendências; 1 na Universidade Federal do Maranhão (UFMA) e 1 na Universidade Federal do Rio Grande do Norte (UFRN). Cabe mencionar que não foram encontrados estudos na região norte.

Os dados acima descritos vão ao encontro das estatísticas disponíveis na Plataforma Lattes - $\mathrm{CNPq}$, por meio dos registros do Diretório dos Grupos de Pesquisa no Brasil, o qual descreve que há predominância de Grupos de Pesquisa (GP) nas regiões sul e sudeste, o que pode justificar o grande número de estudos desenvolvidos nessas regiões, não obstante, a distribuição dos GP por grande área de conhecimento é predominantemente sobre as ciências humanas e da saúde, contemplando a enfermagem. Esses registros corresponderam ao período de 1993 a $2016^{35}$.

No que concerniu ao tipo de abordagem metodológica das teses e dissertações, $23(85,2 \%)$ utilizaram o método quantitativo $^{9-16,18,20-30,32-34}$ e 4 (14,8\%), o método quantitativo-qualitativo (misto $)^{8,17,19,31}$. A análise dos dados predominante foi a estatística descritiva e inferencial, representando 19 (70,4\%) produções ${ }^{8,10,11,14,16,20-32,34}$, seguida de estatística descritiva, somente, com $4(14,8 \%)^{12,13,15,33}$. Após, a estatística inferencial, somente, com $2(7,4 \%)^{9,18}$ e, por fim, $1(3,7 \%)$ estudo utilizou a estatística descritiva junto com a observação não participante ${ }^{17}$ e 1 (3,7\%), a estatística descritiva, inferencial e análise de conteúdo de Bardin ${ }^{19}$.

Essa predominância de método quantitativo pode ser justificada pelo motivo das pesquisas terem utilizado, ao menos, um instrumento validado para a coleta de dados, característica típica desta abordagem. O delineamento quantitativo pode ser representado pela epidemiologia, área que busca levantar dados, frequências, indicadores e prevalências de determinado problema ou doença ${ }^{36}$.

Ainda, houve o domínio das tendências para a análise estatística, que pode ser descritiva ou analítica, o que também representou estudos quantitativos, pois buscou testar hipóteses e associações entre variáveis de interesse. A análise descritiva visa à obtenção do valor preciso dos parâmetros de uma população; já a analítica, observa as características dos elementos de uma amostra e estima o valor do parâmetro, ou a decisão de rejeição ou não de uma hipótese sobre o mesmo ${ }^{36}$.

Quanto aos instrumentos utilizados para a coleta de dados, apareceu em destaque o Questionário Atitudes de Segurança (SAQ) em $16(59,2 \%)$ tendências ${ }^{9,12-14,16,17,19-21,24,26,27,30,32-34}$, seguido 
do questionário Hospital Patiente Safety Culture (HSOPSC), com $8(29,7 \%)^{10,11,15,22,23,25,28,31}$. Ainda, em $2(7,4 \%)$ produções foram utilizados o SAQ, mais o questionário semiestruturado ${ }^{8,18}$, e $1(3,7 \%)$ estudo aplicou o questionário HSOPSC, o instrumento Lista de Sinais e Sintomas (LSS) e o Índice de Satisfação Profissional (ISP) 29 .

No que se referiu ao cenário das tendências, 11 (40,8\%) foram desenvolvidas em todo o ambiente hospitalar ${ }^{10,20,22,24,26-28,31-34}$; $3(11,1 \%)$, em Unidade de Terapia Intensiva Adulto (UTI) ${ }^{14,25,29}$; $2(7,4 \%)$, em UTI neonatal, pediátrica e materno-infantil ${ }^{12,23}$;
$2(7,4 \%)$, em ambiente cirúrgico ${ }^{9,30} ; 2$ (7,4\%), em hospital oncológico ${ }^{8,13}$ e 1 (3,7\%), no centro obstétrico e alojamento conjunto $^{21}$. Também, 1 (3,7\%) estudo foi realizado em uma unidade de transplante renal ${ }^{11} ; 1(3,7 \%)$, em instituição cardiovascular ${ }^{16}$; $1(3,7 \%)$, em unidades de emergência pediátricas ${ }^{15} ; 1$ (3,7\%), em clínica cardíaca ${ }^{17} ; 1$ (3,7\%), em centro de pesquisas oncológicas ${ }^{18} \mathrm{e}$ $1(3,7 \%)$, em cenário acadêmico ${ }^{19}$. Esses cenários dominantes corroboraram com a proposta dos instrumentos utilizados, uma vez que estes foram adaptados e traduzidos para a realidade brasileira para serem desenvolvidos em ambientes hospitalares ${ }^{8-34}$.

Quadro 1: Quadro sinóptico. Produções selecionadas, acerca da temática do estresse e cultura de segurança. Santa Maria, RS, 2017

\section{Título}

Cultura de segurança do paciente: a percepção do enfermeiro em um hospital oncológico Avaliação da cultura de segurança em unidades cirúrgicas

Cultura de segurança do paciente em serviços de enfermagem hospitalar

Cultura de segurança do paciente na ótica da enfermagem em uma unidade de transplante renal Cultura e clima de segurança do paciente em uma maternidade-escola: percepção dos profissionais de enfermagem de terapia intensiva

A cultura de segurança do paciente na perspectiva do enfermeiro

Análise da cultura da segurança entre os profissionais da unidade de terapia intensiva adulto de uma instituição de ensino

Cultura de segurança do paciente em unidades de emergência pediátrica: perspectiva da equipe de enfermagem

Cultura de segurança do paciente na perspectiva da equipe de enfermagem de uma instituição cardiovascular

O cuidar de si como uma dimensão da cultura de segurança do paciente

Cultura de segurança do paciente em unidade catarinense de transplante de medula óssea

A cultura de segurança do paciente na ótica dos acadêmicos de enfermagem

A cultura de segurança do paciente em um hospital de ensino de Minas Gerais

Cultura de segurança do paciente na perspectiva de profissionais da enfermagem obstétrica e neonatal

Análise da cultura de segurança em um hospital de ensino da região centro-oeste do Brasil

Cultura de segurança do paciente em unidades de terapia intensiva neonatal

Cultura de segurança do paciente: percepções e atitudes dos trabalhadores nas instituições hospitalares de Santa Maria

Cultura de segurança do paciente: avaliação dos profissionais de saúde de uma unidade de terapia intensiva

Clima de segurança entre trabalhadores de enfermagem em unidades de internação adulto

Cultura de segurança: avaliação das atitudes de segurança da equipe de enfermagem de um hospital geral de grande porte de Porto Alegre

Avaliação da cultura de segurança do paciente em ambiente hospitalar: estudo comparativo em hospital universitário e público do Rio de Janeiro e do Porto

Segurança do paciente em unidades de terapia intensiva: fatores dos pacientes, estresse, satisfação profissional e cultura de segurança na ocorrência de eventos adversos

Avaliação da cultura de segurança entre profissionais do centro cirúrgico de um hospital de porte especial: elementos para a melhoria da qualidade do cuidado aos idosos submetidos a procedimentos cirúrgicos

Percepção da equipe multiprofissional sobre segurança do paciente: análise sistêmica dos riscos na assistência que podem gerar eventos adversos não infecciosos

Percepção do clima de segurança do paciente em instituições hospitalares

Avaliação do clima de segurança do paciente em Unidade de Emergência de um hospita universitário do interior de São Paulo

Clima de segurança: percepção dos profissionais de enfermagem em um hospital de ensino

\section{Autor, Ano e \\ referência}

Carrera, $2013^{8}$

Cauduro, $2013^{9}$

Costa, $2014^{10}$

Câmara, 2016"11

Fernandes, $2014^{12}$

Matiello, $2015^{13}$

Gomides, $2016^{14}$

Macedo, $2015^{15}$

Misiak, 2013

Gasperi, 2013 ${ }^{17}$

Fermo, 2014 ${ }^{18}$

Ilha, 2014 ${ }^{19}$

Luiz, $2013^{20}$

Batista, 2015²

Tobias, $2013^{22}$

Tomazoni, $2013^{23}$

Baratto, 2015²4

Minuzzi, $2014^{25}$

Nauderer, $2015^{26}$

Souza, 2016 ${ }^{27}$

Fassarella, $2016^{28}$

Ducci, $2015^{29}$

Carvalho, 2014 ${ }^{30}$

Serrano, $2015^{31}$

Mikael, $2016^{32}$

Rigobello, $2015^{33}$

Tondo, $2015^{34}$
Nível acadêmico e Instituição

Dissertação - UNIRIO

Dissertação - UFPR

Dissertação - USP/ Ribeirão Preto

Dissertação - UFMA

Dissertação - UFRN

Dissertação - UFES

Dissertação - UFG

Dissertação - UFSC

Dissertação - UFSC

Tese - UFSC

Dissertação - UFSC

Dissertação - UFSC

Dissertação - UFTM

Dissertação - UFSC

Dissertação - UFG

Dissertação - UFSC

Dissertação - UFSM

Dissertação - UFSC

Tese - FURG

Dissertação - UNISINOS

Tese- UERJ

Tese - USP

Dissertação - FEPECS

Dissertação - UFVJM

Dissertação - USP/

Ribeirão Preto

Dissertação - USP/

Ribeirão Preto

Dissertação - UNICAMP

FEPECS: Escola Superior de Ciências da Saúde; FURG: Universidade Federal de Rio Grande; UERJ: Universidade Estadual do Rio de Janeiro; UFES: Universidade Federal do Espírito Santo; UFG: Universidade Federal de Goiás; UFMA: Universidade Federal do Maranhão; UFPR: Universidade Federal do Paraná; UFRN: Universidade Federal do Rio Grande do Norte; UFSC: Universidade Federal de Santa Catariana; UFSM: Universidade Federal de Santa Maria; UFTM: Universidade Federal do Triângulo Mineiro; UFVJM: Universidade Federal dos Vales do Jequitinhonha e do Mucuri; UNICAMP: Universidade Estadual de Campinas; UNIRIO: Universidade Federal do Estado do Rio de Janeiro; UNISINOS: Universidade do Vale do Rio dos Sinos; USP: Universidade de São Paulo. 
Com relação aos participantes dos estudos, 11 (40,8\%) aplicaram suas pesquisas na equipe de enfermagem ${ }^{10-12,15-17,21,26,27,31,34}$;

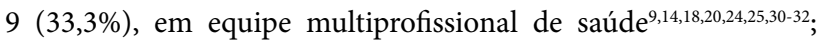
4 (14,8\%), em enfermeiros somente ${ }^{8,13,22,28} ; 1$ (3,7\%), em enfermeiros e médicos ${ }^{23} ; 1(3,7 \%)$, em equipe multiprofissional de saúde e nas equipes de higiene/limpeza e de secretariado ${ }^{33}$ e 1 (3,7\%), em estudantes de enfermagem ${ }^{19}$. Essas populações estudadas contemplam os objetivos dos instrumentos SAQ e HSOPSC, já que buscaram, de maneira geral, avaliar a segurança do paciente, do ambiente e a ocorrência de incidentes na assistência prestada, características que podem ser respondidas pelos profissionais de saúde, visto que estes estavam em contato direto com os pacientes em seu ambiente de trabalho ${ }^{10,13,17}$.

\section{Tendências acerca da gerência, estresse ocupacional e cultura punitiva na enfermagem: fragilidades encontradas}

A fragilidade da cultura de segurança é identificada nos estudos voltados à segurança do paciente. O tema apareceu nas produções, apontando as vulnerabilidades nas ações desenvolvidas e a necessidade de implementar estratégias que contemplassem e colaborassem para uma assistência em saúde com maior qualidade $e^{8,12,14,16}$.

O estresse e as condições de trabalho apareceram com baixos escores nas respostas dos participantes pesquisados, valores de escores que foram considerados inadequados, conforme os parâmetros dos instrumentos utilizados nas pesquisas. Embora os participantes estivessem satisfeitos com o seu trabalho (domínio e satisfação no trabalho), estes apontaram como fragilidades, por exemplo, o quadro de pessoal insuficiente e o fato de ter que trabalhar mais do que o desejado ${ }^{10,11,21,22,26,27,30}$. Esse fator esteve evidenciado nos estudos e associado à ocorrência de incidentes, o que comprometeu a segurança do paciente ${ }^{37}$. Também, o estresse ocupacional foi reconhecido, por parte dos profissionais, como influência negativa no trabalho ${ }^{38}$.

Nesse enfoque, a satisfação profissional e o estresse ocupacional estiveram relacionados, entre si, uma vez que a não realização no trabalho associada aos estressores do ambiente laboral repercutiram na cultura de segurança ${ }^{29}$. Assim, tanto o estresse ocupacional quanto a insatisfação no trabalho estavam relacionadas com as atitudes e os comportamentos de segurança dos profissionais para o desempenho de suas atividades ${ }^{29,38,39}$.

Um estudo que objetivou identificar o nível de estresse em enfermeiros que atuavam em cuidados diretos ao paciente crítico, a partir da avaliação do nível de estresse no contexto hospitalar no transcorrer de suas atividades, evidenciou maior nível de estresse ocupacional associado à assistência prestada ao paciente. Também, o mesmo estudo identificou que o estresse ocupacional estava associado a atividades relacionadas à administração de pessoal e às condições de trabalho para o desempenho das atividades do enfermeiro ${ }^{40}$.

Outra tendência prevalente com baixos escores nos resultados dos estudos foi com relação à percepção dos profissionais frente à gerência das unidades, fator este sendo avaliado negativamente, de maneira geral, pelos participantes, os quais desaprovavam as ações da gerência quanto às questões de segurança e a necessidade de maior investimento por parte da gestão ${ }^{13,14,16,18,26-28,30,32-34}$ Nesse domínio, estudos apontaram a falta de apoio diário da administração dos hospitais com seus funcionários como fator negativo para a cultura de segurança ${ }^{38,39}$.

Corroborando, alguns estudos destacaram a existência da cultura punitiva e de culpa, evidenciando a necessidade de mudança cultural relacionada à abordagem do erro humano ${ }^{11,23,28}$. Nesse sentido, as gerências das unidades, junto às instituições como um todo, precisariam criar estratégias que proporcionassem uma boa atmosfera no ambiente de trabalho, para que questões como estas pudessem ser discutidas em diálogo aberto, contemplando um ambiente não punitivo ${ }^{39}$.

Vale ressaltar que algumas produções apontaram que a cultura de segurança estava em constante consolidação, apresentando potenciais forças de melhorias nas dimensões assistenciais, o que pôde possibilitar a criação de estratégias específicas para cada setor de saúde ${ }^{25,31}$. Essas melhorias podem estar relacionadas com os esforços dos órgãos e instituições na busca por uma assistência cada vez mais qualificada. Assim, o entendimento da dimensão que corresponde à cultura de segurança tem evoluído consideravelmente ${ }^{37}$.

Nessa perspectiva, cabe a reflexão a respeito dos estressores laborais e as suas implicações para a cultura de segurança, visto que se trata de uma temática em consolidação e que necessita de novas investigações. Com isso, será possível obter subsídios que venham a favorecer a saúde do trabalhador, por meio da redução do estresse ocupacional e, consequentemente, favorecer a cultura de segurança do paciente.

Por fim, considerou-se como limitações deste estudo a busca ter sido realizada apenas no Portal de teses e dissertações da CAPES, visto que algumas universidades têm seus próprios reservatórios e não disponibilizam os estudos na plataforma. Ainda, alguns resumos apresentaram poucas informações, o que dificultou a apresentação dos resultados e discussão.

\section{CONSIDERAÇÕES FINAIS}

Desvendou-se, como uma tendência das produções, a predominância dos estudos de cultura de segurança em unidades fechadas. Percebeu-se que as teses e as dissertações buscaram estudar outros fatores relacionados com a cultura de segurança, além do estresse ocupacional. A insatisfação profissional e a falta de recursos materiais e humanos surgiram como estressores ocupacionais, desfavorecendo a cultura de segurança.

As produções, em um panorama geral, apontaram fatores que serviram de alerta para a cultura de segurança, tais como, a percepção do estresse ocupacional, por parte dos profissionais de saúde, a carga de trabalho elevada, a cultura de punição e culpa, fragilidade na segurança do paciente e ações incipientes, por parte 
da gerência das unidades. Outros fatores identificados foram a escassez de estudos, com abordagem qualitativa e a ausência de estudos na temática da cultura de segurança associada ao estresse ocupacional no contexto da atenção primária em saúde. Essas características podem servir para que sejam desenvolvidas investigações que preencham as lacunas encontradas.
Vale ressaltar que algumas produções não puderam ser analisadas, por não apresentarem resumo, ou por este estar incompleto, o que pode ser considerado um fator limitante do presente estudo. Em contraponto, obteve-se a oportunidade de conhecer as principais produções da temática, o que contribuiu para a construção do conhecimento em saúde e enfermagem.

\section{REFERÊNCIAS}

1. Institute of Medicine (US) Committee on Quality of Health Care in America; Kohn LT, Corrigan JM, Donaldson MS. To err is human: building a heath system. Washington (DC): National Academies Press (US); 2000.

2. Carvalho REFL, Cassiani SHB. Questionário de Atitudes de Segurança: adaptação transcultural do Safety Attitudes Questionnaire - Short Form, 2006 para o Brasil. Rev Latino-Am Enfermagem. 2012;20(3):575-82. http://dx.doi.org/10.1590/S0104-11692012000300020

3. Karasek RA. Job demands, job decision latitude, and mental strain: implications for job redesign. Adm. Sci. Q. 1979;24:285-308.

4. Andrade RVS, Costa ORS. Estresse ocupacional em profissionais de saúde: um estudo com a equipe de enfermagem da Unidade de Terapia Intensiva - UTI de um Hospital Escola em Minas Gerais. Rev Ciênc Saúde. 2014;4(4):1-11. http://dx.doi.org/10.21876/rcsfmit.v4i4.261

5. Silva EFL, Moura MLC. Estresse nas relações enfermeiro/paciente: revisão integrativa. Rev Enferm UFPE. 2014;8(7):2140-8. http://dx.doi.org/10.5205/reuol.5963-51246-1-RV.0807201440

6. Matiello RDC, Lima EFA, Coelho MCR, Oliveira ERA, Leite FMC, Primo CC. A cultura de segurança do paciente na perspectiva do enfermeiro. Cogitare Enferm. 2016;21(esp):1-9. http://dx.doi.org/10.5380/ce.v21i5.45408

7. Rother ET. Revisão sistemática $x$ revisão narrativa. Acta Paul. Enferm. 2007; 20(2):v-vi. http://dx.doi.org/10.1590/S0103-21002007000200001

8. Carrera AS, Aguiar BG. Cultura de segurança do paciente: a percepção do enfermeiro em um hospital oncológico. Dissertação (Mestrado) - Universidade Federal do Estado do Rio de Janeiro. Rio de Janeiro: 2013.

9. Cauduro FLF. Avaliação da cultura de segurança em unidades cirúrgicas. Dissertação (Mestrado) - Universidade Federal do Paraná. Curitiba: 2013.

10. Costa DB. Cultura de segurança do paciente em serviços de enfermagem hospitalar. Dissertação (Mestrado) - Universidade de São Paulo, Escola de Enfermagem de Ribeirão Preto. Ribeirão Preto: 2014.

11. Câmara JJC. Cultura de segurança do paciente na ótica da enfermagem em uma unidade de transplante renal. Dissertação (Mestrado) - Universidade Federal do Maranhão. São Luís: 2016.

12. Fernandes LGG. Clima e cultura de segurança do paciente em uma maternidade escola: percepção dos profissionais de enfermagem em terapia intensiva. Dissertação (Mestrado) Universidade Federal do Rio Grande do Norte. Natal: 2014.

13. Matiello RDC. A cultura de segurança do paciente na perspectiva do enfermeiro. Dissertação (Mestrado) - Universidade Federal do Espírito Santo. Vitória: 2015.

14. Gomides M. Análise da cultura da segurança entre os profissionais da unidade de terapia intensiva adulto de uma instituição de ensino. Dissertação (Mestrado) - Universidade Federal de Goiás. Catalão: 2016.

15. Macedo M. Cultura de segurança do paciente em unidades de emergência pediátrica: perspectiva da equipe de enfermagem. Dissertação (Mestrado) - Universidade Federal de Santa Catarina. Florianópolis: 2015

16. Misiak M. Cultura de segurança do paciente na perspectiva da equipe de enfermagem de uma instituição cardiovascular. Dissertação (Mestrado) - Universidade Federal de Santa Catarina. Florianópolis: 2013.

17. Gasperi P. O cuidar de si como uma dimensão da cultura de segurança do paciente. Tese (Doutorado) - Universidade Federal de Santa Catarina. Florianópolis: 2013.

18. Fermo VC. Cultura de segurança do paciente em unidade catarinense de transplante de medula óssea. Cultura de segurança do paciente em unidade catarinense de transplante de medula óssea. Dissertação (Mestrado) - Universidade Federal de Santa Catarina. Florianópolis: 2014.

19. Ilha P. A cultura de segurança do paciente na ótica dos acadêmicos de enfermagem. Dissertação (Mestrado) - Universidade Federal de Santa Catarina. Florianópolis: 2014.

20. Luiz RB. A cultura de segurança do paciente em um hospital de ensino de Minas Gerais. Dissertação (Mestrado) - Universidade Federal do Triângulo Mineiro. Uberaba: 2013.

21. Batista ACO. Cultura de segurança do paciente na perspectiva de profissionais da enfermagem obstétrica e neonatal. Dissertação (Mestrado) - Universidade Federal de Santa Catarina. Florianópolis: 2015

22. Tobias GC. Análise da cultura de segurança em um hospital de ensino da região centro-oeste do Brasil. Dissertação (Mestrado) Universidade Federal de Goiás. Goiânia: 2013.

23. Tomazoni A. Cultura de segurança do paciente em unidades de terapia intensiva neonatal. Dissertação (Mestrado) - Universidade Federal de Santa Catarina. Florianópolis: 2013.

24. Baratto MAM. Cultura de segurança do paciente: percepções e atitudes dos trabalhadores nas instituições hospitalares de Santa Maria. Dissertação (Mestrado) - Universidade Federal de Santa Maria. Santa Maria: 2015.

25. Minuzzi AP. Cultura de segurança do paciente: avaliação dos profissionais de saúde de uma unidade de terapia intensiva. Dissertação (Mestrado) - Universidade Federal de Santa Catarina. Florianópolis: 2014

26. Nauderer TM. Clima de segurança entre trabalhadores de enfermagem em unidades de internação adulto. Tese (Doutorado) - Universidade Federal do Rio Grande. Rio Grande: 2015.

27. Souza AB. Cultura de segurança: avaliação das atitudes de segurança da equipe de enfermagem de um hospital geral de grande porte de Porto Alegre. Dissertação (Mestrado) Universidade do Vale do Rio dos Sinos. São Leopoldo: 2016. 
28. Fassarella CS. Avaliação da cultura de segurança do paciente em ambiente hospitalar: estudo comparativo em Hospital Universitário e público do Rio de Janeiro e do Porto. Tese (Doutorado) Universidade do Porto. Porto: 2016.

29. Ducci AJ. Segurança do paciente em Unidades de Terapia Intensiva: fatores dos pacientes, estresse, satisfação profissional e cultura de segurança na ocorrência de eventos adversos. Tese (Doutorado) - Universidade de São Paulo. São Paulo: 2015

30. Carvalho PA. Avaliação da cultura de segurança entre profissionais do centro cirúrgico de um hospital de porte especial: elementos para a melhoria da qualidade do cuidado aos idosos submetidos a procedimentos cirúrgicos. Dissertação (Mestrado) - Fundação de Ensino e Pesquisa em Ciências da Saúde. Brasília: 2014

31. Serrano ACFF. Percepção da equipe multiprofissional sobre segurança do paciente: análise sistêmica dos riscos na assistência que podem gerar eventos adversos não infecciosos. Dissertação (Mestrado) - Universidade Federal dos Vales do Jequitinhonha e Mucuri. Diamantina: 2015.

32. Mikael SSE. Percepção do clima de segurança do paciente em instituições hospitalares. Dissertação (Mestrado) - Universidade de São Paulo, Escola de Enfermagem de Ribeirão Preto. Ribeirão preto: 2016.

33. Rigobello MCG. Avaliação do clima de segurança do paciente em Unidade de Emergência de um hospital universitário do interior de São Paulo. Dissertação (Mestrado) - Universidade de São Paulo, Escola de Enfermagem de Ribeirão Preto. Ribeirão Preto: 2015.
34. Tondo JCA. Clima de segurança: percepção dos profissionais de enfermagem em um hospital de ensino. Dissertação (Mestrado) Universidade Estadual de Campinas, Faculdade de Enfermagem. Campinas: 2015.

35. Diretório dos Grupos de Pesquisa no Brasil. Plataforma Lattes/ CNPq. Disponível em: http://lattes.cnpq.br/web/dgp. Acesso em: 15 maio 2017

36. Turato ER. Métodos qualitativos e quantitativos na área da saúde: definições, diferenças e seus objetos de pesquisa. Rev Saúde Pública. 2005;39(3):507-14.

http://dx.doi.org/10.1590/S0034-89102005000300025

37. Novaretti MCZ, Santos EV, Quitério LM, Daud-Gallotti RM. Sobrecarga de trabalho da Enfermagem e incidentes e eventos adversos em pacientes internados em UTI. Rev Bras Enferm. 2014;67(5):692-9. http://dx.doi.org/10.1590/0034-7167.2014670504

38. Correggio TC, Amante LN, Barbosa SFF. Avaliação da cultura de segurança do paciente em centro cirúrgico. Rev SOBECC. 2014;19(2):67-73

http://dx.doi.org/10.4322/sobecc.2014.012

39. Rigobello MCG, Carvalho REFL, Cassiani SHB, Galon T, Capucho HC, Deus NN. Clima de segurança do paciente: percepção dos profissionais de enfermagem. Acta Paul Enferm. 2012;25(5):728-35 http://dx.doi.org/10.1590/S0103-21002012000500013

40. Almeida AMO, Lima AKG, Vasconcelos MGF, Soares Lima AC Oliveira GYM. Estresse ocupacional em enfermeiros que atuam em cuidados ao paciente crítico. Rev Enferm UFPE. 2016:10(5):1663-71. http://10.5205/reuol.9003-78704-1-SM.1005201612 